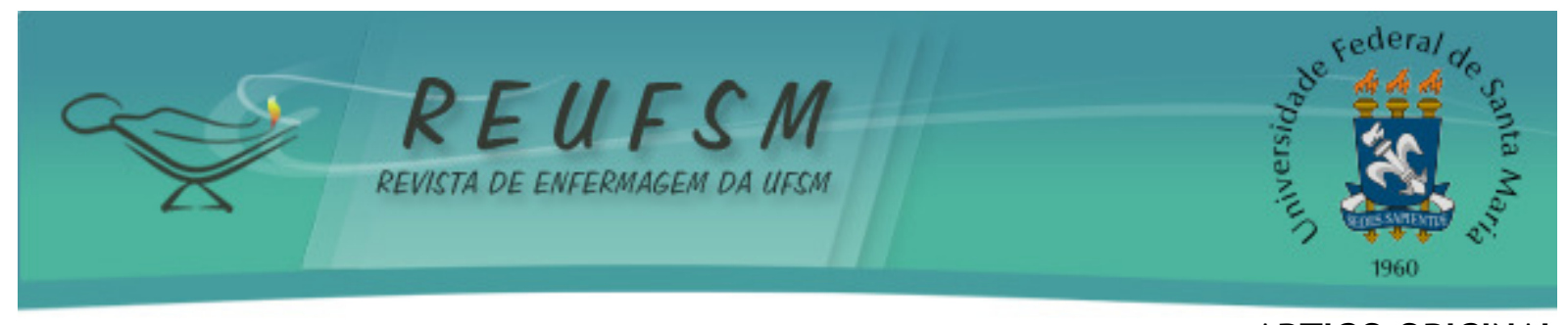

ARTIGO ORIGINAL

\title{
CARACTERIZAÇÃO DA MORBIMORTALIDADE DE RECÉM NASCIDOS INTERNADOS EM UNIDADE DE TERAPIA INTENSIVA NEONATAL*
}

\section{CHARACTERIZATION OF MORBIDITY AND MORTALITY NEWLY BORN IN HOSPITALIZED NEONATAL INTENSIVE CARE UNIT}

\section{CARACTERIZACIÓN DE LA MORBIMORTALIDAD DE LOS RECIÉN NACIDOS EN UNA UNIDAD DE CUIDADO INTENSIVO NEONATAL}

\author{
Andrea Moreira Arrué \\ Eliane Tatsch Neves ${ }^{2}$ \\ Andressa da Silveira ${ }^{3}$ \\ Greice Machado Pieszak ${ }^{4}$
}

RESUMO: Objetivo: caracterizar a morbimortalidade dos recém-nascidos (RN) internados em uma Unidade de Terapia Intensiva Neonatal do sul do Brasil, no ano de 2004. Método: estudo documental, descritivo, retrospectivo. A população foi composta por 302 neonatos. A coleta de dados foi realizada diretamente nos prontuários, utilizando-se um formulário próprio, incluindo variáveis relacionadas à realização de pré-natal, motivo da internação, morbidades e causas de óbito. Os dados quantitativos foram submetidos à análise estatística descritiva. Resultados: tem-se a prematuridade como o principal motivo de internação e causa de óbito. Em relação à morbidade destacaram-se síndrome do desconforto respiratório do RN, distúrbios hematológicos e infecção neonatal. Conclusões: as afecções relacionadas à gestação e nascimento foram os principais motivos de internações. Recomendam-se um acompanhamento pré-natal e pós-natal de qualidade, intervenções qualificadas no parto e na assistência ao RN no intuito de diminuir os índices de morbimortalidade neonatal.

Descritores: Terapia intensiva neonatal; Mortalidade neonatal; Morbidade; Recém-nascido; Enfermagem neonatal.

ABSTRACT: Objective: To characterize the mortality of newborns (NB) in a Neonatal Intensive Care Unit in southern Brazil, in 2004. Method: documentary, descriptive, retrospective study. The population consisted of 302 neonates. Data collection was performed directly in the medical records, using a form own, including variables related to were conducting pre-natal care, reason for hospitalization, morbidity y causes of death. Quantitative data were descriptive statistically analyzed. Results: have to prematurity as the main reason for hospitalization and cause of death. Regarding morbidity highlights the respiratory distress syndrome of the newborn, hematological disorders and neonatal infection. Conclusions: the listed conditions during pregnancy and

\footnotetext{
${ }^{1}$ Enfermeira. Mestre em Enfermagem pelo Programa de Pós-Graduação em Enfermagem (PPGEnf) da Universidade Federal de Santa Maria (UFSM/RS). Membro do Grupo de Pesquisa Cuidado às Pessoas, Famílias e Sociedade -PEFAS. E-mail: andrea.mor@hotmail.com

${ }^{2}$ Enfermeira. Doutora em Enfermagem. Professora Adjunta do Departamento de Enfermagem e do Programa de Pós-Graduação em Enfermagem de Santa Maria (UFSM/RS). Membro do Grupo de Pesquisa Cuidado às Pessoas, Famílias e Sociedade - PEFAS. E-mail: elianeves03@gmail.com

${ }^{3}$ Enfermeira. Mestre em Enfermagem. Professora Assistente do Departamento de Enfermagem da Universidade Federal do Pampa (UNIPAMPA). Membro do Grupo de Pesquisa Cuidado às Pessoas, Famílias e Sociedade PEFAS. Vice-líder do Grupo de Pesquisa GEPEnf - FORS. Coordenadora do PROEXT/MEC Práticas Integradas em Saúde Coletiva (PISC). Tutora do PET Redes na área de Atenção Básica. E-mail: andressadasilveira@gmail.com ${ }^{4}$ Enfermeira. Mestre em Enfermagem pelo Programa de Pós-Graduação em Enfermagem (PPGEnf) da Universidade Federal de Santa Maria (UFSM/RS). Membro do Grupo de Pesquisa Cuidado às Pessoas, Famílias e Sociedade -PEFAS. E-mail: greicepieszak@gmail.com
} 


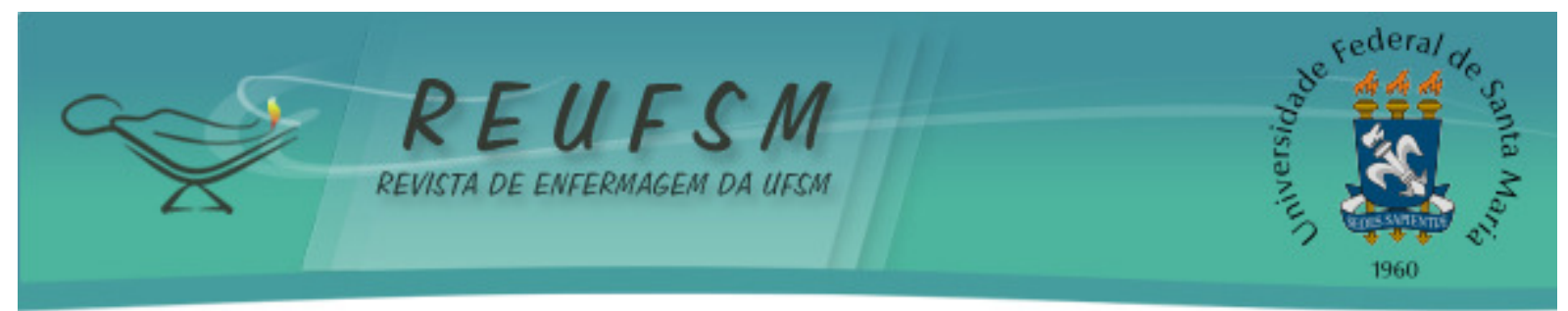

birth were the main cause of hospital admissions. We recommend a prenatal and postnatal quality, skilled assistance at delivery and in assisting the RN in order to reduce the rates of morbidity and mortality.

Descriptors: Intensive care; Neonatal mortality; Morbidity; Infant; Neonatal nursing.

RESUMEN: Objetivo: caracterizar la morbimortalidad de los recién nacidos (RN) internados en una Unidad de Cuidados Intensivos Neonatales en Sur de Brasil, en 2004. Método: estudio documental, descriptivo, retrospectivo. La población fue constituida por $302 R N$. La recolección de datos se realizó directamente en las fichas clínicas, utilizando un formulario propio, incluidas variables relacionadas con realización de la atención prenatal, razón por la hospitalización, morbilidades y causas de muerte. Los datos cuantitativos fueron analizados utilizando estadística descriptiva. Resultados: la prematuridad como principal razón para hospitalización y causa de muerte. En relación a la morbilidad destaca síndrome de dificultad respiratoria del $R N$, trastornos hematológicos e infección neonatal. Conclusiones: las condiciones que se indican durante embarazo y parto son la principal causa de ingresos hospitalarios. Se recomienda una calidad prenatal y postnatal, la asistencia calificada durante el parto y en la asistencia al $R N$ con el fin de reducir las tasas de morbimortalidad.

Descriptores: Cuidado intensivo neonatal; Mortalidad neonatal; Morbilidad; Recién nacido; Enfermería neonatal.

\section{INTRODUÇÃO}

No Brasil a taxa de mortalidade infantil (TMI) encontra-se em declínio, apresentando uma redução de $20,5 \%$ entre os anos de 1996 e 2000 e de $27 \%$ entre 2000 e 2007. ${ }^{1}$ Além disso, as causas da mortalidade infantil alteraram-se nas últimas décadas, reduzindo-se as causas infectocontagiosas e elevando-se as causas perinatais, que são decorrentes de problemas durante a gestação, parto e nascimento.

No Rio Grande do Sul, nos anos de 2008 e 2009, os condicionantes de óbitos de menores de um ano totalizaram $27,3 \%$ de doenças congênitas ou anomalias, $23 \%$ de afecções geradas no período perinatal, $12,5 \%$ de doenças respiratórias e 7,4\% de mortes por doenças infecciosas ou parasitárias. ${ }^{2}$ Em contrapartida à queda na TMI, tem-se as morbidades por doenças congênitas e atreladas ao período perinatal, em ritmo crescente.

Pesquisa realizada em um hospital de ensino para verificar as demandas de crianças com necessidades especiais de saúde ${ }^{3}$ constatou que em $58,5 \%$ dos casos o surgimento da necessidade especial de saúde estava diretamente relacionada às causas perinatais, incluindo aquelas associadas às condições de parto e nascimento. Portanto, destaca-se a importância da qualidade no atendimento à criança no período neonatal (zero a 27 dias), pois é considerado um período crítico que representa maior risco diário de morte. ${ }^{4}$ Além disso, os óbitos neonatais são resultantes de uma estreita e complexa relação de fatores sociodemográficos, condições da gestação e do parto, bem como das características dos prematuros nascidos vivos. ${ }^{1,5}$

A morbimortalidade neonatal em países subdesenvolvidos e em desenvolvimento é determinada principalmente, pelos diversos fatores de risco relacionados com as condições gerais de vida e o acesso aos serviços de saúde. O mérito fica a cargo da precariedade do atendimento e da oferta de serviços públicos, tornando fatores evitáveis de comprometimento perinatal a sua grande causa. ${ }^{6}$

O Brasil vem avançando na redução da mortalidade infantil, os índices de mortalidade pós-natal tiveram grande decréscimo com a implantação de programas de prevenção e promoção de saúde materno-infantil, mas ainda é preciso um grande esforço 


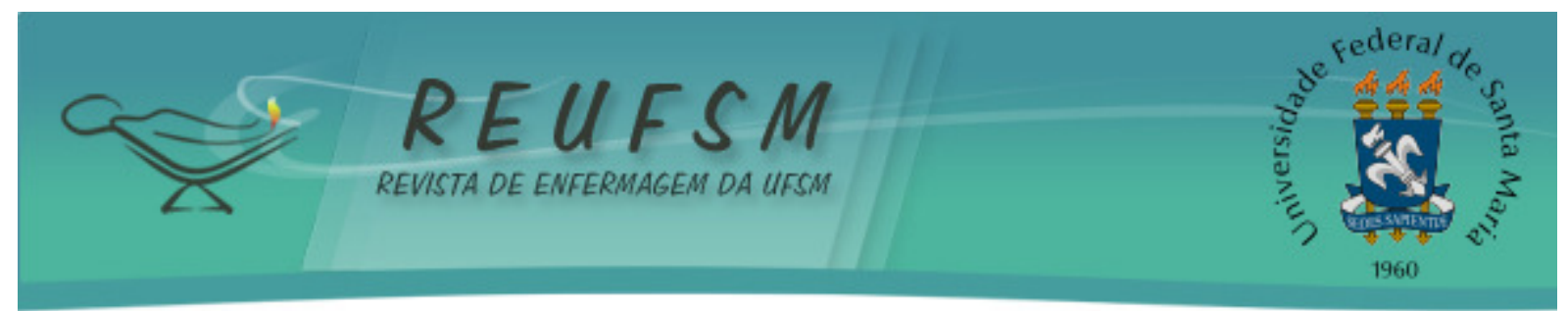

para enfrentar as diferenças regionais e alcançar patamares aceitáveis. ${ }^{7}$ A redução da mortalidade neonatal representa um desafio para os serviços de saúde, uma vez que a maioria dessas mortes poderia ser evitada se houvesse melhores condições na atenção prénatal e na assistência ao parto. ${ }^{8}$

Desse modo, para enfrentar este desafio é necessário o conhecimento dos fatores predisponentes a morbimortalidade neonatal como subsídio para (re) estruturar a assistência, (re)direcionar e subsidiar ações de acordo com as especificidades e prioridades desse grupo.

Partindo dessas reflexões, questionou-se qual é o índice de morbimortalidade dos neonatos admitidos em uma UTIN. Este estudo teve por objetivo caracterizar a morbimortalidade dos recém-nascidos internados em uma UTIN do sul do Brasil, no ano de 2004.

\section{MÉTODO}

Trata-se de uma análise documental quantitativa, descritiva de caráter retrospectivo que foi desenvolvida a partir do recorte da pesquisa intitulada: "Caracterização da morbimortalidade e das demandas de necessidades especiais de saúde dos recém-nascidos internados em uma UTI Neonatal no sul do Brasil”.

O hospital cenário do estudo caracteriza-se como de alta complexidade, referência no atendimento em saúde para toda a Região Central do Rio Grande do Sul. Possui a única Unidade de Tratamento Intensivo Neonatal (UTIN) pública da região, que recebe uma média de 30 crianças mensalmente, totalizando cerca de 350 internações/ano.

A população de estudo foram os 302 neonatos internados na UTIN do Hospital Universitário, procedentes de Santa Maria (RS), no ano de 2004. Os critérios de exclusão foram: peso inferior a 500 gramas e/ou idade gestacional inferior a 24 semanas, uma vez que esses dados são considerados como aborto. ${ }^{8}$ Também foram excluídos pacientes portadores de anomalias congênitas incompatíveis com a vida e/ou transferidos para outras instituições. Além de prontuários que não informavam o peso ao nascer e/ou idade gestacional e/ou prontuários com informações incompletas que não possibilitassem a caracterização do RN.

A coleta de dados foi realizada diretamente nos prontuários, no período entre abril de 2008 a agosto de 2010. Foi utilizado um formulário próprio previamente testado, incluindo variáveis relacionadas à gestação, nascimento, parto e internação na Unidade de Terapia Intensiva Neonatal (UTIN), os dados estavam disponíveis no arquivo da instituição.

Para o processamento eletrônico e pré-análise, os dados foram codificados, digitados em planilha do programa Excel e submetidos aos aplicativos de análise estatística descritiva no System Analyses Statistics (SAS) e PAlaeontological STatistics (PAST) $1.34 .{ }^{9} 0$ estudo foi aprovado pelo Comitê de Ética em Pesquisa da instituição (CEP/UFSM), sob número: 0233.0.243.000-08.

\section{RESULTADOS E DISCUSSÃO}

Em relação à morbidade e a mortalidade dos RNs internados na UTIN procedentes de Santa Maria, no ano de 2004, constatou-se que a prematuridade e as complicações durante o parto foram prevalentes. As infecções, os distúrbios respiratórios e as insuficiências renais, foram decorrentes, na maioria das vezes, de prolongados períodos de internação e uso de tecnologias invasivas, para manter a estabilidade da criança.

No que tange ao sexo do RNs $58 \%$ eram masculino e $42 \%$ feminino. As principais intercorrências apresentadas na hora do parto foram cianose (34\%), desconforto 


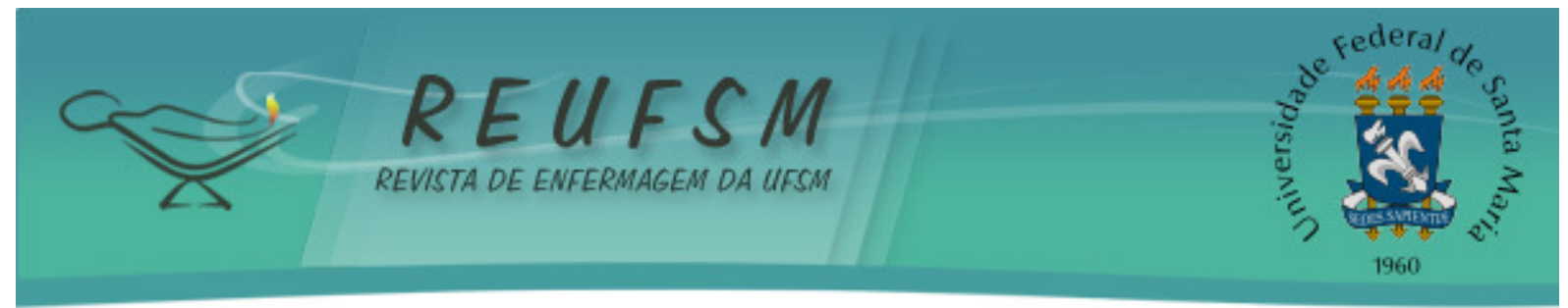

respiratório (25\%) e hipotonia (20\%). Na Tabela 1, será exposto o motivo da internação do RN na UTIN.

TABELA 1: Motivo da internação dos RNs, admitidos em uma UTIN, procedentes de Santa Maria, no ano de 2004. Brasil, RS, $2013(\mathrm{~N}=302)$

\begin{tabular}{ccc}
\hline Motivo da Internação & Frequência(N) & (\%) \\
\hline Prematuridade & $\mathbf{1 7 2}$ & $\mathbf{5 7}$ \\
Desconforto Respiratório & $\mathbf{1 7 2}$ & $\mathbf{5 7}$ \\
Baixo peso & 29 & 10 \\
Distúrbios Hematológicos & 29 & 10 \\
Distúrbios Gastrointestinais & 16 & 5 \\
Distúrbios Neurológicos & 12 & 4 \\
\hline
\end{tabular}

Pode-se constatar que a prematuridade e o desconforto respiratório foram as principais causas das internações neonatais. Os RNs muitas vezes apresentaram mais de uma causa de internação perfazendo uma frequência de 445 motivos. Em estudo semelhante, no Sul do Brasil, em uma UTIN de um Hospital Geral $47 \%$ dos neonatos internaram por prematuridade. ${ }^{10}$

A idade gestacional e o baixo peso ao nascer determinam os prognósticos da prematuridade, devido à relevância na maturidade de vários sistemas em recém-nascido prematuros. ${ }^{11}$ Assim, a prematuridade é uma adversidade devido às altas taxas de mortalidade e pelos riscos que pode acarretar no desenvolvimento dos recém-nascidos.

A prematuridade, como morbidade, está diretamente relacionada aos distúrbios respiratórios e às complicações infecciosas e neurológicas. ${ }^{12}$ Durante o período de internação os neonatos desenvolveram algumas morbidades, dentre elas destaca-se o Desconforto Respiratório ( $n=204)$, os distúrbios hematológicos $(n=140)$ e infecção $(n=126)$. Destaca-se que alguns neonatos desenvolveram mais de uma morbidade, ao longo da internação, representando concomitantemente 664 patologias.

Os fatores neonatais associados à prematuridade estão diretamente relacionados aos distúrbios respiratórios e às complicações infecciosas e neurológicas. ${ }^{12}$ A prevalência de morbidades e complicações neonatais em uma UTIN em Minas Gerais mostrou que a principal complicação e/ou morbidade foi à infecção congênita ou neonatal $(96,5 \%){ }^{13}$

No estudo, a mortalidade neonatal teve uma incidência de $8,6 \%$ entre os RNS da UTIN, procedentes de Santa Maria, no ano de 2004. Em pesquisa realizada no Rio Grande do Sul que analisou os determinantes da mortalidade neonatal evidenciou-se que 0 coeficiente de mortalidade neonatal foi $8,2 \%{ }^{14}$

A prematuridade com $68 \%$ representou a maior causa de óbito, seguida por parada cardiorrespiratória $47 \%$ e septicemia $36 \%$. Grande parte dos óbitos ocorreu pelo somatório dessas patologias.

Apesar dos avanços da perinatologia nos últimos anos, a prematuridade continua sendo a principal causa de morbidade e mortalidade neonatal, representando um dos maiores desafios para o fornecimento de uma assistência profissional de qualidade. ${ }^{12}$

O tempo de internação dos RNs variou de acordo com o Gráfico 1: 

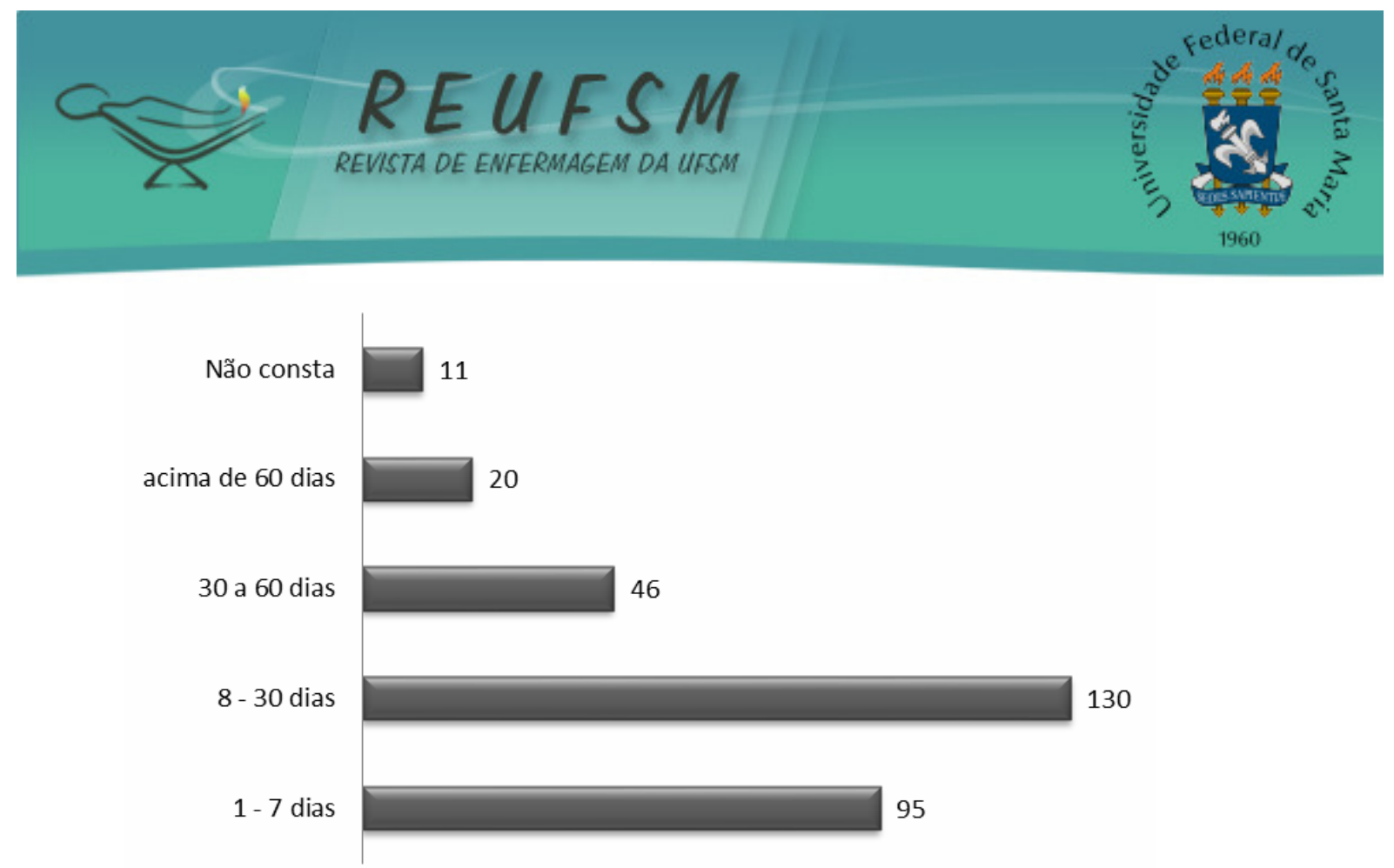

GRÁFICO 1: Tempo de internação dos RNs admitidos em uma UTIN, procedentes de Santa Maria, no ano de 2004. Brasil, RS, $2013(\mathrm{~N}=302)$

A permanência na internação mostrou-se mais frequente no período de 8 a 30 dias. 0 tempo de hospitalização mostrou-se significativo quando relacionado à prematuridade, ou seja, quanto maior for o tempo de internação menor a idade gestacional. ${ }^{13}$

Com relação às consultas de pré-natal constatou-se que $86 \%(n=223)$ das gestantes realizaram acompanhamento, no entanto, $14 \%(n=35)$ não compareceram a nenhuma consulta. Destaca-se que este dado não constava em 44 prontuários. Além disso, não havia registro nos prontuários sobre a qualidade desse acompanhamento bem como o número de consultas. Em estudo, realizado no Rio Grande do Sul que avaliou as variáveis maternas constatou que $72 \%$ das mulheres realizaram consulta de pré-natal e $28 \%$ de nenhuma a três consultas. $^{15}$

Salienta-se que o aumento no número de consultas pré-natais está diretamente relacionado, com a diminuição do retardo no crescimento intrauterino, prematuridade, baixo peso e óbito por essas causas. ${ }^{10}$ Nesse sentido, ressalta-se a importância da realização de exames de urina durante a gestação, pois a infecção do trato urinário na gestante é uma forte predisposição para o parto prematuro e morbidades para o RN. ${ }^{16}$

Um maior número de consultas no pré-natal funciona como fator protetor para o óbito neonatal, indicando a importância dos cuidados durante a gestação. Assim, um acompanhamento gestacional mais assíduo pode identificar precocemente e prevenir ocorrências lesivas para o recém-nascido. ${ }^{17}$ Logo, a garantia de uma assistência de qualidade à gestante possibilita identificar e intervir previamente, reduzindo os riscos e danos causados a saúde do neonato.

\section{CONCLUSÕES}

Quanto à morbidade e a mortalidade dos RNs internados na UTIN no ano de $2004 \mathrm{em}$ Santa Maria, RS, prevaleceram a prematuridade e as complicações durante o parto. A prematuridade foi o principal fator de risco e representou o maior índice de óbito entre os recém-nascidos procedentes da localidade. Os achados permitiram concluir, também, que o tempo de hospitalização mostrou-se significativo quando relacionado à prematuridade.

A cianose foi a principal intercorrência apresentada na hora do parto e, em relação ao motivo de internação dos RNs destacam-se o desconforto respiratório e a 


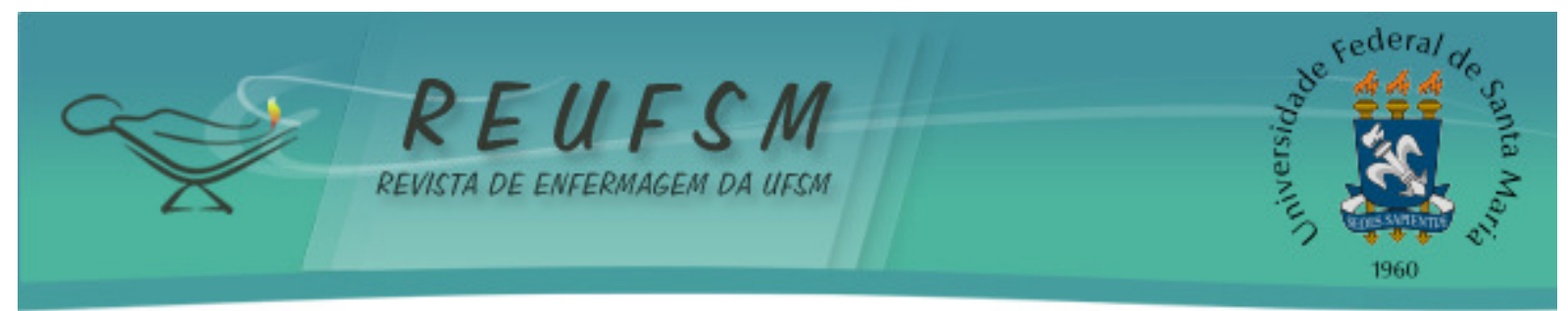

prematuridade. Além disso, o estudo evidenciou a predominância do sexo masculino entre os internados na UTIN.

Os longos períodos de internação e o uso de técnicas e tecnologias avançadas e invasivas estiveram relacionados com as principais morbidades desenvolvidas durante a internação, entre elas: os distúrbios respiratórios, hematológicos e as infecções neonatais.

Os achados permitiram concluir, também, que se o RN interna na UTIN por prematuridade extrema pode evoluir rapidamente ao óbito ou necessitar de longo período de internação. Esta constatação possibilita refletir sobre a qualidade da assistência desenvolvida na UTIN que pode ocasionar seqüelas na qualidade de vida desta criança no pós-alta hospitalar.

O estudo concluiu, ainda, que a maioria das gestantes realizaram pré-natal. Portanto, recomenda-se enfatizar a qualidade dos programas pré-natais, incentivando o acompanhamento da gestação o mais cedo possível. Isto poderia minimizar os riscos de complicações durante o parto, ou pelo menos prevenir que este aconteça em caráter emergencial. Sugerem-se, também, investimentos na qualificação da equipe multidisciplinar considerando que a recepção do recém-nascido é um momento crucial e que pode ter repercussões na vida futura da criança.

\section{REFERÊNCIAS}

1. DATASUS - Departamento de Informática do Sistema Único de Saúde. Nascidos vivos. Indicadores e dados básicos - IDB 2009. [Internet]. Brasília (DF): Ministério da Saúde [acesso em 2012 jun 29]. Disponível em: http://www.datasus.gov.br/idb.

2. DATASUS - Departamento de Informática do Sistema Único de Saúde. Nascidos vivos. Óbitos Infantis no Rio Grande do Sul. [Internet]. Brasília (DF): Ministério da Saúde [acesso em 2012 jun 29]. Disponível em: http:/ / tabnet.datasus.gov.br/cgi/deftohtm.exe?sim/cnv/inf10rs.def.

3.Vernier ETN, Cabral IE. Caracterização de crianças com necessidades especiais de saúde e seus familiares cuidadores. Santa Maria (RS). 2004-2005: subsídios para intervenção de enfermagem. Rev Sociedade Brasileira Enfermeiros Pediatras. São Paulo [Internet]. 2006 [acesso em 2012 jun 10];6(1):37-45. Disponível em: http://www.sobep.org.br/revista/2006-volume-6/numero-1.html.

4. Zanini RR, Soares AB, Giugliani ERJ, Riboldi J. Determinantes da mortalidade neonatal no RS. Rev Saúde Pública. 2011;45(1):79-89.

5. Ramos HAC, Cuman RKN. Fatores de risco para prematuridade: pesquisa documental. Esc Anna Nery Rev Enferm. 2009;13(2):297-304.

6. Soares ES, Menezes GMS. Fatores associados à mortalidade neonatal precoce: análise da situação no nível local. Epidemiol Serv Saúde. 2010 jan-mar;19(1):51-60.

7. Brasil. Ministério da Saúde. Secretaria de Atenção à Saúde. Manual de vigilância do óbito infantil e fetal e do comitê de prevenção do óbito infantil e fetal. Brasília: Editora do Ministério da Saúde; 2009. 77 p. (Série A. Normas e Manuais Técnicos).

8. Rocha R, Oliveira C, Silva DKF, Bonfim C. Mortalidade neonatal e evitabilidade: uma análise do perfil epidemiológico. Rev Enferm UERJ [Internet]. 2011 jan-mar [acesso em 2012 jun 2];19(1):114-20. Disponível em: http://www.facenf.uerj.br/v19n1/v19n1a19.pdf.

9. Hammer O, Harper DAT, Ryan PD. PAST - Palaeontological Statistics. Versão 1.34. 2003. 


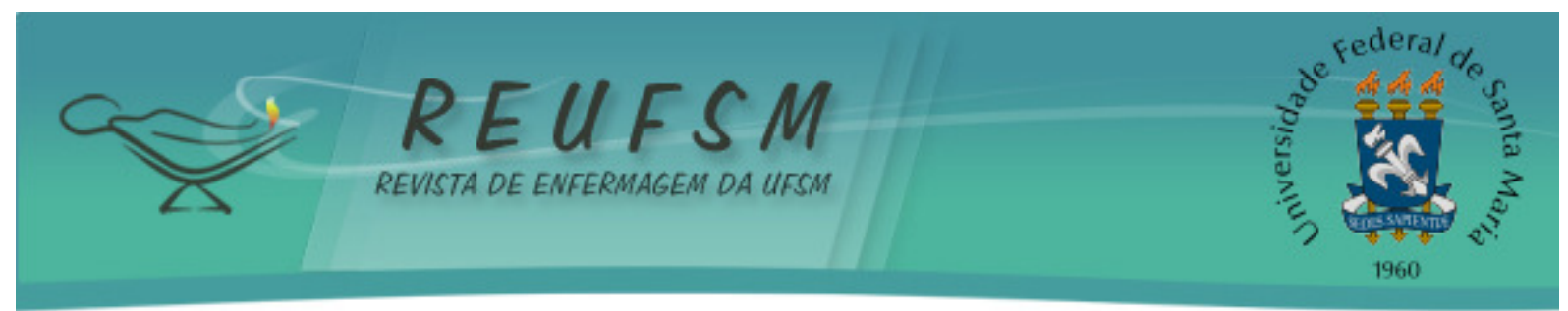

10. Araújo BF, Tanaka ACA, Madi JM, Zatti H. Estudo da mortalidade de recém-nascidos internados na UTI neonatal do Hospital Geral de Caxias do Sul, Rio Grande do Sul. Rev Bras Saúde Matern Infant. 2005;5(4):463-9.

11. Andreani G, Custódio ZAO, Crepaldi MA. Tecendo as redes de apoio na prematuridade. Aletheia [Internet]. 2006 dez [acesso em 2012 jun 30];(24):115-26. Disponível em: http: / / pepsic.bvsalud.org/scielo.php?script=sci_arttext\&pid=S1413-

$03942006000300011 \&$ ing=pt.

12. Salge AKM, et al. Fatores maternos e neonatais associados à prematuridade. Rev Eletr Enf [Internet]. 2009 [acesso em 2012 jun 18];11(3):642-6. Disponível em: http://www.fen.ufg.br/revista/v11/n3/v11n3a23.htm.

13. Lemos RA, Frônio JS, Neves LAT, Ribeiro LC. Estudo da prevalência de morbidades e complicações neonatais segundo o peso ao nascimento e a idade gestacional em lactentes de um serviço de follow-up. Rev APS. 2010;13(3):277-90.

14. Zanini RR, Moraes AB, Giugliani ERJ, Riboldi J. Determinantes contextuais da mortalidade neonatal no Rio Grande do Sul por dois modelos de análise. Rev Saúde Pública. 2011;45(1):79-89.

15. Padilha JF, Farinha LB, Mattos KM, Gasparetto A. Caracterização da saúde materna em Santa Maria, RS. 2005-2009. Rev Enferm UFSM [Internet]. 2012 jan/abr [acesso em 2012 jun 30];2(1):79-87. Disponível em: http://cascavel.ufsm.br/revistas/ojs2.2.2/index.php/reufsm/article/view/4082/3129.

16. Silveira MF, Barros AJD, Santos IS, Matijasevich A, Victora CG. Diferenciais socioeconômicos na realização de exame de urina no pré-natal. Rev Saúde Pública. 2008;42(3):389-95.

17. Soares ES, Menezes GMS. Fatores associados à mortalidade neonatal precoce: análise de situação no nível local. Epidemiol Serv Saúde [Internet]. 2010 mar [acesso em 2012 jul 10];19(1):51-60. Disponível

http: //scielo.iec.pa.gov.br/scielo.php?script=sci_arttext\&pid=S1679-

49742010000100007\&lng=pt.

Data de recebimento: $26 / 07 / 2012$

Data de aceite: 02/05/2013

Contato com autora responsável: Andrea Moreira Arrué

Endereço postal: Rua Arariboia, n²73. Bairro São João. Volta Redonda/RJ. CEP: 27252-340

E-mail: andrea.mor@hotmail.com 